\title{
Spatial and Temporal Distribution of Soil Water Content in Fields under Different Vegetation Conditions Based on TDR Measurements
}

\author{
Teruhito MIYAMOTO ${ }^{1 *}$, Mahithon PUTISO ${ }^{2}$, Takahiro SHIONO ${ }^{1}$, \\ Hiroyuki TARUYA ${ }^{1,4}$ and Jiro CHIKUSHI ${ }^{3}$ \\ ${ }^{1}$ Department of Agro-Environmental Research, National Agricultural Research Center for Kyushu \\ Okinawa Region (Nishigoshi, Kumamoto 861-1192, Japan) \\ ${ }^{2}$ Land Development Department, Ministry of Agriculture and Co-operatives Thailand \\ (Mittraparp Road, Khon Kaen 40000, Thailand) \\ ${ }^{3}$ Biotron Institute, Kyushu University (Hakozaki, Fukuoka 812-8581, Japan)
}

\begin{abstract}
TDR (Time-domain reflectometry) is an advanced method for monitoring the water content in fields TDR measurements can provide accurate information about vegetation effects on both spatial and temporal soil water distribution. The objective of this study was to examine the effects of vegetation conditions on the distribution of the soil water content. Soil water content was measured to express the spatial distribution in the surface layer $(0-0.2 \mathrm{~m})$ in grass, corn, and non-planted fields. Additionally, using the TDR method with multiple probes with different lengths, the measurements were conducted on a temporal basis at 4 depths $(0-0.1,0.1-0.2,0.2-0.3$ and $0.3-0.5 \mathrm{~m})$ to determine the soil-water content profiles in the 3 fields. A significantly higher water content in both the grass and cornfields was found, compared to the non-planted field. Moreover, in the cornfield, we observed that microtopographic features significantly affected the spatial water distribution. The lower the soil water content, the higher the coefficient of variation of the soil moisture content. These results indicated that the effects of the vegetation conditions on the soil water distribution in the fields were significant. From the observation of the temporal soil water profiles in the 3 fields, it was found that soil management, i.e. tillage or no-tillage, was a major factor affecting the profiles.
\end{abstract}

Discipline: Agricultural engineering

Additional key words: electromagnetic wave, Andisol, multiple probes with different lengths

\section{Introduction}

Monitoring soil water content is important for managing regional water resources appropriately. Water balances in soils are influenced considerably by the vegetation conditions in the field. However, limited information is available regarding the spatial distribution of the soil water content under different cropping regimes. To evaluate the effect of these differences on soil water distribution, measurements of soil water profiles must be conducted simultaneously and continuously at locations where the environmental conditions are similar except for vegetation.

The Time-Domain Reflectometry (TDR) method, which is the most advanced technique for measuring the soil water content, has become increasingly popular worldwide. Since the TDR method is an accurate, rapid and non-destructive method for measuring the soil water content in fields, it is suitable for measuring the spatial distribution of the water content of the surface soil ${ }^{8,10}$. Moreover, as one of the variations in the installation of the TDR probe, Topp and Davis (1985) introduced a technique using multiple probes with different lengths to measure the soil moisture profile 9 . In this technique, TDR probes with different lengths were vertically inserted into soils and the soil water content at a specific depth was estimated based on the difference in the travel times along those probes. By applying this method to a field, the wetting front patterns in soil were determined

Present address:

${ }^{4}$ Department of Hydraulic Engineering, National Institute for Rural Engineering (Tsukuba, Ibaraki 305-8609, Japan)

*Corresponding author: fax +81-96-249-1002; e-mail teruhito@affrc.go.jp

Received 29 August 2003; accepted 20 October 2003. 
under 2 different irrigation methods ${ }^{7}$. It was also verified that the method is useful for measuring the soil water distribution under different tillage systems ${ }^{3}$.

The objective of our study was to examine the effects of vegetation conditions on the distribution of the soil water content. To this end, we applied the TDR method with multiple probes with different lengths for measuring the spatial and temporal distribution of the soil water content in fields under different vegetation conditions, i.e. corn, grass, and non-planted fields.

\section{Materials and methods}

\section{Water content measurement}

Although the principle of the TDR method has been described elsewhere ${ }^{1,2,9}$, we will briefly outline it. In the TDR method, the relationship between the water content and apparent dielectric permittivity $\left(\varepsilon_{a}\right)$ for moist soils is used. Values of dielectric permittivities are around 81 for water, 3-5 for most mineral soil components, and 1 for air. Due to this large disparity in the values of dielectric permittivity, the TDR method appears to be relatively more sensitive to soil water than to other components. Therefore, the relationship between the volumetric water content $(\theta)$ and $\varepsilon_{a}$ must be estimated prior to the measurement of the soil water content.

To measure the water content in a given layer, TDR probes were embedded in soil as shown in Fig. 1. In general, the propagation velocity, $v$, of an electromagnetic wave along the TDR probe with length, $L$, (travel distance $2 L$ ) embedded in soil is determined from the signal travel time, $t$, using the TDR cable tester $(v=2 L / t)$. The dielectric permittivity can be given by the equation:

$$
\varepsilon_{a}=\left(\frac{c}{v}\right)^{2}=\left(\frac{c t}{2 L}\right)^{2}
$$

where $c$ is the propagation velocity of an electromagnetic wave in free space $\left(3 \times 10^{8} \mathrm{~m} \mathrm{~s}^{-1}\right)$.

In Fig. 1, the measured layer corresponds to the difference between the 2 rod lengths, $L 1$ and $L 2$. If the travel times $t_{1}$ and $t_{2}$ are assumed for $L 1$ and $L 2$, respectively, the difference between the TDR probe lengths being $(2 \times(L 2-L 1))$, then the average apparent dielectric permittivity between the lengths $L 1$ and $L 2, \bar{\varepsilon}$, is:

$$
\bar{\varepsilon}=\left[\frac{L 2 \times\left(\varepsilon_{2}\right)^{1 / 2}-L 1 \times\left(\varepsilon_{1}\right)^{1 / 2}}{(L 2-L 1)}\right]^{2}
$$

where $\varepsilon_{1}$ and $\varepsilon_{2}$ are the apparent dielectric permittivities determined from the propagation velocities along the TDR probes with length $L 1$ and $L 2$, respectively. Based

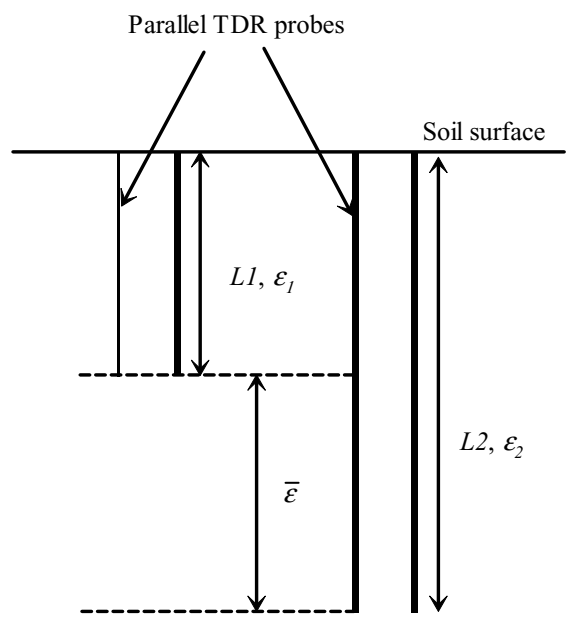

Fig. 1. Design of TDR measurement using multiple probes with different lengths

$\varepsilon_{1}$ and $\varepsilon_{2}$ are the apparent dielectric permittivities determined from the propagation velocities along the TDR probes with length $L 1$ and $L 2$, respectively. $\bar{\varepsilon}$ is the average apparent dielectric permittivity between length $L 1$ and $L 2$.

on the calibrated relationship between $\varepsilon_{a}$ and $\theta$, the average soil moisture in the range between $L 1$ and $L 2$ depths can be determined from $\bar{\varepsilon}$ obtained by Eq. (2).

\section{Field experiments}

Field experiments were carried out at the National Agricultural Research Center for Kyushu Okinawa Region in Kumamoto, Kyushu, Japan. We prepared 3 different plots, a corn plot, a grass plot and a non-planted plot, $2 \times 40 \mathrm{~m}$ in size. The upslope of the grass plot was $1.6 \%$, while the corn plot and the non-planted plot were almost flat. These plots were located at $32^{\circ} 53^{\prime}$ North Latitude and $130^{\circ} 45^{\prime}$ East Longitude within a distance of $1 \mathrm{~km}$, and the soil in these plots was an Andisol (Hydric Pachic Melanudand). In the corn plot, the soil was plowed to a depth of $0.15-0.20 \mathrm{~m}$ and corn (Zea mays L.) seeds were sown on April 5, 2002, with a row width of $0.80 \mathrm{~m}$. In the grass plot, the soil had not been tilled for more than 14 years. Bahiagrass (Paspalum notanum Flugge) was grown and harvested in August and October each year using heavy machinery. In contrast to these plots, in the non-planted plot, the soil had been subjected to rotary plowing and tilled in early June 2002.

Field measurements of the spatial distribution of the soil water content were conducted 3 times under different cropping conditions during the period from June 18 to June 29 in 2002. One hundred TDR probes were installed vertically in a single transect, perpendicular to the cropping rows, at $0.4 \mathrm{~m}$ intervals in each of the 3 


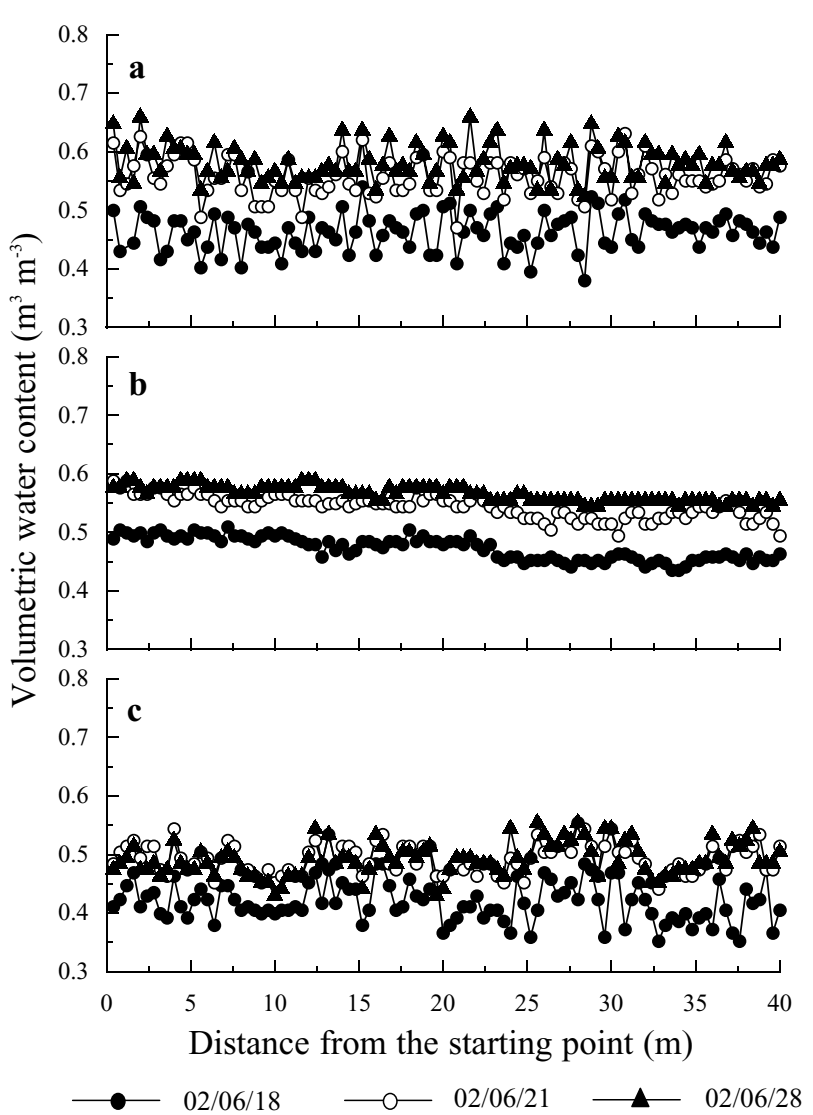

Fig. 2. Spatial distribution of soil surface water along the transect a: Corn plot. b: Grass plot. c: Non-planted plot.

plots. Each probe consisted of 2 stainless steel transmission rods ( $3 \mathrm{~mm}$ in diameter, $0.2 \mathrm{~m}$ in length, $0.05 \mathrm{~m}$ in width). The measurement depth $(0.2 \mathrm{~m})$ corresponded to the depth of tillage in the corn plot. The TDR measurements were conducted using a portable cable tester (Tektronix Model 1502B) connected to the TDR probes through a balun. The balun had an impedance transformer to match the impedance of the coaxial and shielded antenna cable. The TDR trace on the cable tester was used to calculate $\varepsilon_{a}$ and associated soil water content for each probe using the $\varepsilon_{a}-\theta$ relationship for Andisols $^{3}$ :

$$
\begin{aligned}
\theta= & -0.0356+4.35 \times 10^{-2} \varepsilon_{a}-1.23 \times 10^{-2} \varepsilon_{a}^{2} \\
& +1.48 \times 10^{-5} \varepsilon_{a}^{3}
\end{aligned}
$$

For monitoring the temporal distribution of the soil water content in the different fields, soil water content measurements were carried out for 4 different layers at 3 points in each plot every day from June 18 to June 29 in 2002. The TDR measurement points were $10 \mathrm{~m}$ apart from each other. At each point, TDR probes ( $3 \mathrm{~mm}$ in diameter) with 4 different lengths, $0.10,0.20,0.30,0.50 \mathrm{~m}$ were vertically inserted into the soil. The measurement points in the corn plot were selected in interrows.

The data of the measured water content were analyzed by statistical methods ${ }^{4}$. In addition, semivariograms $\gamma(h)$ of the soil water content were calculated to evaluate the spatial correlation, using the equation:

$$
\gamma(h)=\frac{1}{2 N(h)} \sum_{i=1}^{N(h)}\left[Z\left(x_{i}+h\right)-Z\left(x_{i}\right)\right]^{2}
$$

where $x_{i}$ is the $x$ coordinate of the measurement point, $Z\left(x_{i}\right)$ is the measured value at each point $x_{i}[i=1,2, \ldots$, $N(h)], h$ is lag, and $N(h)$ is the total number of pairs of measurements for given $h$.

\section{Results and discussion}

\section{Spatial distribution of soil water content among corn, grass and non-planted plots}

The spatial distribution of the soil surface water was considerably influenced by the differences in the vegetation conditions (Fig. 2a-2c). The water distribution in 
Table 1. Statistics of soil water content data in plots under 3 different vegetation conditions

\begin{tabular}{|c|c|c|c|c|c|c|c|}
\hline $\begin{array}{l}\text { Plots \& } \\
\text { experimental date }\end{array}$ & Mean & $\mathrm{SD}^{\mathrm{a})}$ & $\mathrm{SE}^{\mathrm{b})}$ & $\mathrm{CV}^{\mathrm{c})}$ & Min. & Max. & $N$ \\
\hline \multicolumn{8}{|l|}{ Non-planted plot } \\
\hline $02 / 06 / 18$ & 0.419 & 0.0330 & 0.0033 & 0.0789 & 0.352 & 0.484 & 100 \\
\hline $02 / 06 / 21$ & 0.493 & 0.0257 & 0.0026 & 0.0522 & 0.441 & 0.554 & 100 \\
\hline $02 / 06 / 28$ & 0.491 & 0.0285 & 0.0028 & 0.0579 & 0.429 & 0.554 & 100 \\
\hline \multicolumn{8}{|l|}{ Corn plot } \\
\hline $02 / 06 / 18$ & 0.462 & 0.0315 & 0.0031 & 0.0681 & 0.380 & 0.540 & 100 \\
\hline $02 / 06 / 21$ & 0.558 & 0.0327 & 0.0033 & 0.0586 & 0.470 & 0.631 & 100 \\
\hline $02 / 06 / 28$ & 0.581 & 0.0311 & 0.0031 & 0.0535 & 0.523 & 0.659 & 100 \\
\hline \multicolumn{8}{|l|}{ Grass plot } \\
\hline $02 / 06 / 18$ & 0.472 & 0.0198 & 0.0020 & 0.0418 & 0.435 & 0.509 & 100 \\
\hline $02 / 06 / 21$ & 0.544 & 0.0198 & 0.0020 & 0.0364 & 0.494 & 0.588 & 100 \\
\hline $02 / 06 / 28$ & 0.565 & 0.0129 & 0.0013 & 0.0228 & 0.544 & 0.588 & 100 \\
\hline \multicolumn{8}{|c|}{ Grass plot (de-trended data) } \\
\hline $02 / 06 / 18$ & 0.472 & 0.0105 & 0.0010 & 0.0222 & 0.448 & 0.501 & 100 \\
\hline $02 / 06 / 21$ & 0.544 & 0.0126 & 0.0013 & 0.0232 & 0.508 & 0.577 & 100 \\
\hline $02 / 06 / 28$ & 0.565 & 0.0072 & 0.0007 & 0.0128 & 0.549 & 0.581 & 100 \\
\hline
\end{tabular}

a): Standard deviation. b): Standard error. c): Coefficient of variation.

the corn plot (CP) exhibited a wave pattern due to microtopography effects such as row and interrow (Fig. 2a). On the other hand, no particular pattern of water distribution was detected in the grass plot (GP) (Fig. 2b). Decrease in the water content was observed along a single transect for moisture patterns in GP. These linear trends of the water content data can be ascribed to the upslope of the plot in this direction $(1.6 \%)$. In the nonplanted plot (NP), the water content largely varied without a specific trend (Fig. 2c).

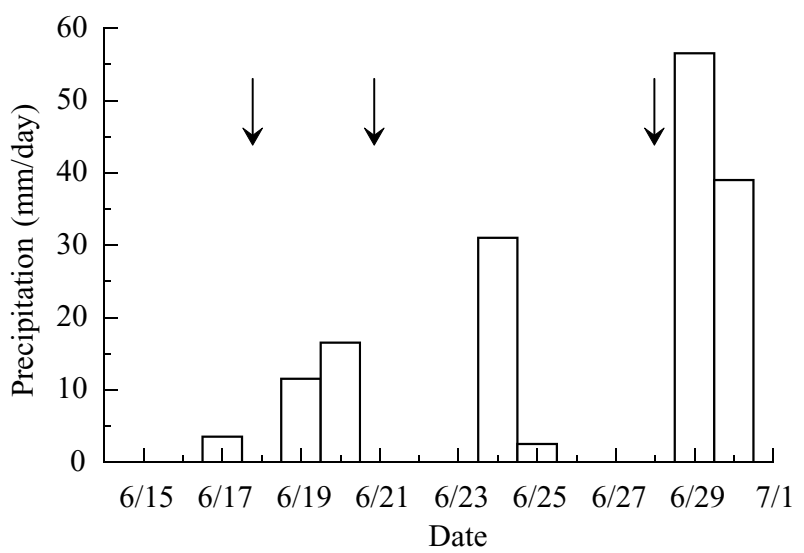

Fig. 3. Precipitation during the observation period (6/15/ 2002-6/30/2002)

Arrows indicate the date when the measurements of the spatial distribution of the soil water content were conducted.
The statistics of the water content data of the 3 plots under different vegetation conditions are summarized in Table 1. The lowest mean values of each plot were recorded on June 18. This result may have been influenced by the rainfall conditions (Fig. 3). The coefficient of variation $(\mathrm{CV})$ of the soil water content changes with the level of the soil water content. That is, the lower the soil water content, the higher the $\mathrm{CV}^{5,8}$.

Comparison of the mean values among the 3 plots indicated that the CP showed the highest soil water content among the 3 plots. The soil water content in NP was always about 5\% lower than that in both CP and GP. This may be due to the fact that grass usually has short and fine roots, leading to a uniform root structure, while corn has coarse and large roots, resulting in a heterogeneous soil structure. For the NP condition, the soil surface layer still retained a large amount of macro-pores after tillage.

These different features of the water content distribution could also be detected in the semivariograms (Fig. 4). The semivariogram for CP fluctuated and could be described by a cosine function. For GP, the range of the semivariogram was relatively wide and seemed to be best expressed by a spherical function. In the semivariogram for NP, the range was narrow compared to that of GP, and could be expressed by a spherical or exponential function $^{6}$. In $\mathrm{CP}$, the spatial correlation was strongly influenced by the micro-topography, while in GP and NP, the spatial pattern of variability was similar but the range of influence was different. 


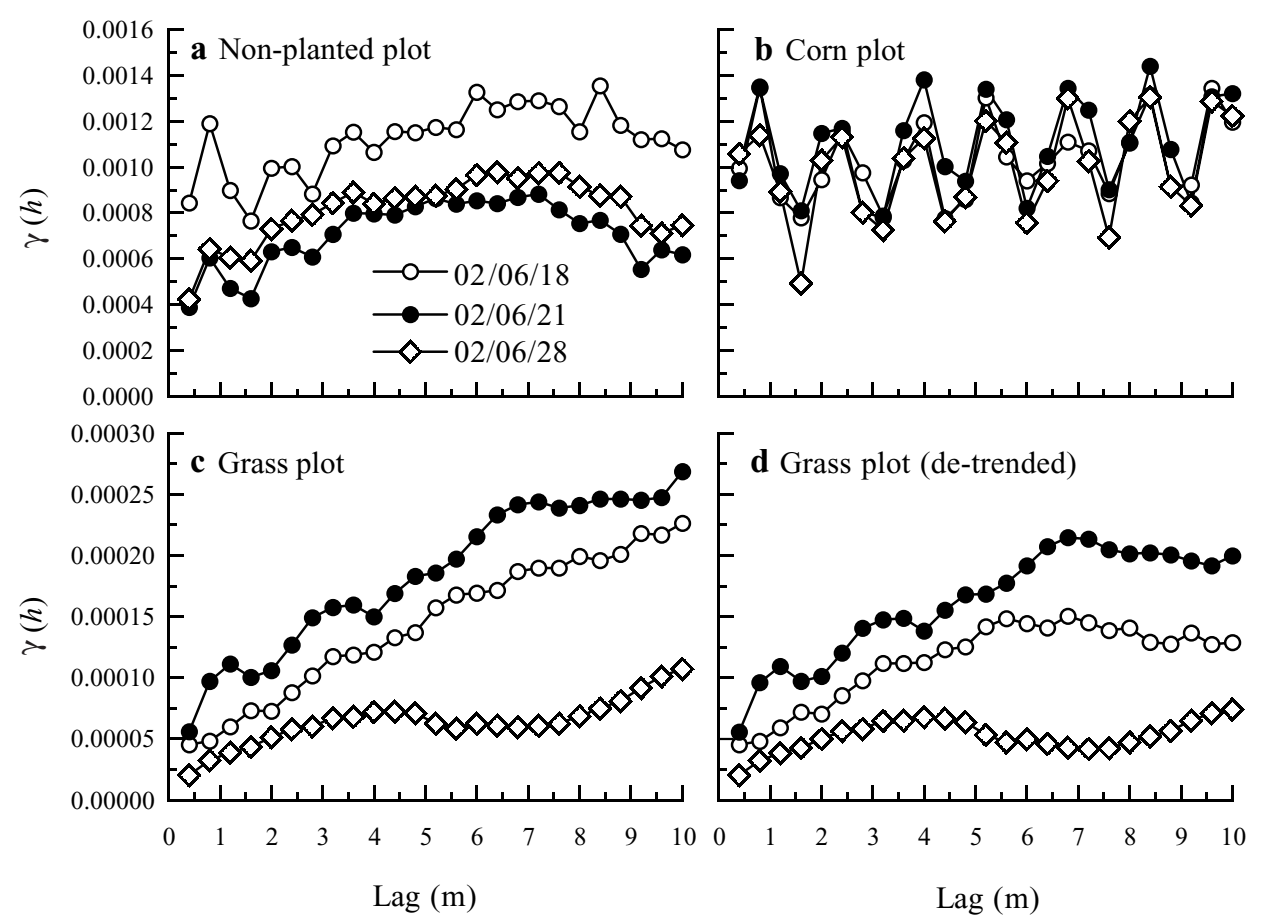

Fig. 4. Semivariograms of soil moisture content in the 3 plots

Semivariograms obtained by using de-trended data in the grass plot are also shown.

The CV of the soil water content in NP showed a similar value to that in $\mathrm{CP}$, and these $\mathrm{CV}$ values were $2-3$ times higher than that of GP, because in $\mathrm{CP}$, soil water distribution can be influenced by the micro-topography, while in NP, heterogeneity of soil properties may lead to a large CV.

\section{Temporal distribution of soil water content among corn, grass and non-planted plots}

Figs. $5 \mathrm{a}-5 \mathrm{c}$ show the changes in the water content in CP, GP, and NP during a 13-day observation period. In $\mathrm{CP}$ and GP, the top layer $(0-0.1 \mathrm{~m})$ contained the largest amount of soil water in the soil profile during the observation period (Fig. 5a, 5b). In contrast to these plots, in $\mathrm{NP}$, the soil water content in the third layer was always the highest during the observation period (Fig. 5c). Moreover, the variations in the soil water content with depth in NP were larger than the variations in the soil water content in both CP and GP. The difference in the soil water profiles between the planted and non-planted plots was most likely caused by the difference in the type of water loss in the profile. Except for drainage loss to the deeper layer, transpiration played a larger role than evaporation in GP and CP. In these plots, soil water can be removed uniformly from the root zone through plant shoot and root systems. The water loss in NP was probably caused by evaporation that reduced the water content mainly in the surface layer.

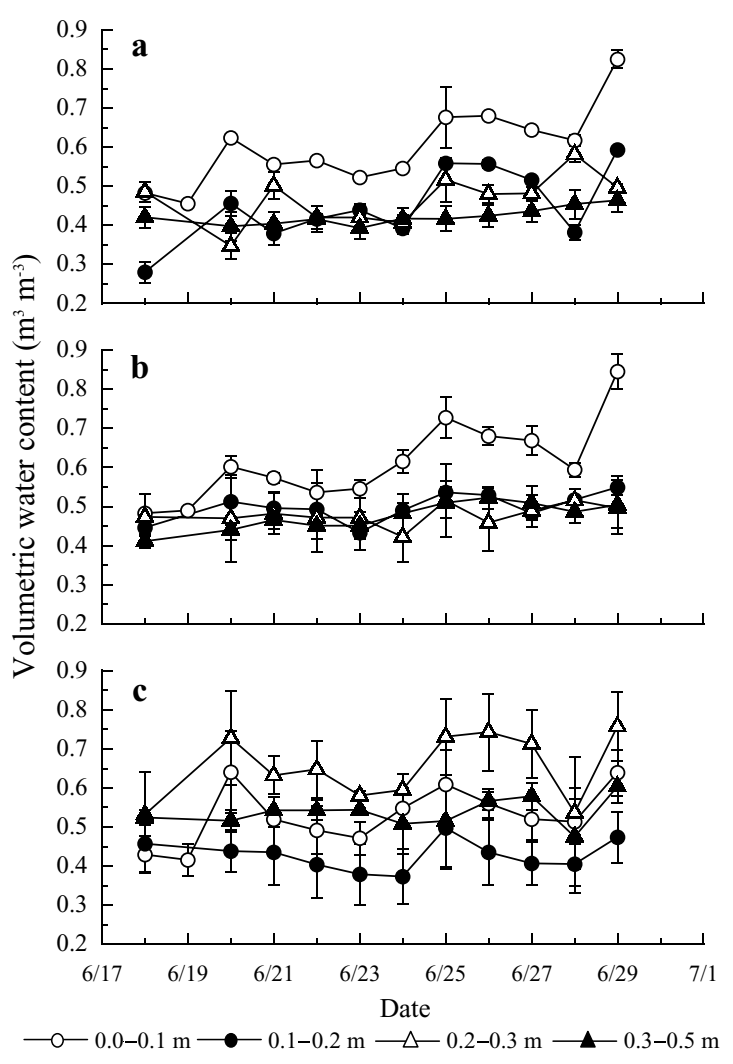

Fig. 5. Time variation in the 4 layer-average soil moisture content in the 3 plots

a: Corn plot. b: Grass plot. c: Non-planted plot. Error bars represent the standard error (SE) of measured data $(n=3)$. 
The changes in the water content in the deeper layers were different between CP and GP. Especially after a $31 \mathrm{~mm}$ rainfall on June 24, the soil water content in both the second and third layers increased in CP (Fig. 5a). On the other hand, the water content remained constant in the second and third layers in GP (Fig. 5b). The difference in the soil water profile between CP and GP was probably due to the difference in soil management. In GP, no-tillage and use of heavy machinery for more than 14 years had led to the formation of a hard soil layer at around 0.1 $m$ depth, hence the decrease in the permeability of the top layer.

\section{Concluding remarks}

Water balances in soils are influenced considerably by the vegetation conditions. We examined the spatial and temporal distribution of the soil water content in fields under 3 different vegetation conditions by using the TDR method. A significantly higher water content in both the grass and cornfields was observed compared to the non-planted field. Moreover, in the cornfield, we found that the micro-topographic features significantly affected the spatial water distribution. It was also observed that in general, the lower the soil water content, the higher the coefficient of variation of the soil water content, and that the soil water content in fields covered with vegetation remained higher than that under the nonplanted condition. Temporal water distribution after rainfall showed different patterns between the cornfield and grass field, that is, the water content in the deeper layer increased in the cornfield, but remained constant in the grass field. These results suggest that cropping condi- tions, soil management, and microtopography strongly affect the soil water distribution in fields. For analyzing these features, the TDR method was found to be very useful.

\section{References}

1. Dalton, F. N. (1992) Development of Time-Domain Reflectometry for measuring soil water content and bulk soil electrical conductivity. In Advances in measurement of soil physics properties: Bringing theory into practice. SSSA Spec. Publ. no. 30, SSSA, USA, 143-167.

2. Dirksen, C. (1999) Soil physics measurements. Catena Verl., Germany, pp. 154.

3. Miyamoto, T. et al. (2001) Applicability of multiple length TDR probes to measure water distributions in an Andisol under different tillage systems in Japan. Soil Tillage Res., 60, 91-99.

4. Miyazaki, T. (1993) Water flow in soils. Marcel Dekker, Inc., New York, pp. 296.

5. Nielsen, D. R., Bigger, J. W. \& Erh, K. T. (1973) Spatial variability of field-measured soil-water properties. Hilgardia, 42, 215-238.

6. Nielsen, D. R. \& Wendroth, O. (2003) Spatial and temporal statistics. Catena Verl., Germany, pp. 398.

7. Pelletier, G. \& Tan, C. G. (1993) Determining irrigation wetting patterns using time domain reflectometry. Hort. Sci., 28, 338-339.

8. Rajkai, K. \& Rydén, B. E. (1992) Measuring areal soil moisture distribution with the TDR method. Geoderma, 52, 73-85.

9. Topp, G. C. \& Davis, J. L. (1985) Measurement of soil water content using time-domain reflectometry (TDR): a field evaluation. Soil Sci. Soc. Am. J., 49, 19-24.

10. van Wesenbeeck, I. J. \& Kachanoski, R. G. (1988) Spatial and temporal distribution of soil water in the tilled layer under a corn crop. Soil Sci. Soc. Am. J., 52, 363-368. 